\title{
Load Analysis on The Bridge Structure According to RSNI T 02-2005 and SNI 1725-2006
}

\author{
Fendy Rachmat ${ }^{1}$,Irpan Hidayat ${ }^{2}$ \\ ${ }^{1}$ Civil Engineering Department, Faculty of Engineering, Bina Nusantara University, Jakarta, Indonesia 11480 \\ ${ }^{2}$ Civil Engineering Department, Faculty of Engineering, Bina Nusantara University, Jakarta, Indonesia 11480, \\ irpan@binus.ac.id
}

\begin{abstract}
The rapid development of infrastructure in civil engineering must be compensated with the latest regulations to adapt to the current conditions, especially in the construction of bridge infrastructure, and therefore the government issued the latest loading regulation on bridge structure that is SNI 1725-2016. The bridge is important because it serves as a liaison between two separate places due to several conditions. This research is conducted to find out whether existing bridge can still meet the criteria of stress, deflection, and deformation based on load value factor, load action, and load combination on SNI 1725-2016. The earthquake load used is a response spectrum using SNI 2833: 2013 regulations[1]. This research uses finite element method using software midas civil software program. In this analysis it is found that the value of stress, deflection, and deformation on the bridge structure according to SNI 17252016 is greater than RSNI T 02-2005.
\end{abstract}

Key words :Bridge, Deflection, Deformation, Voltage

\section{INTRODUCTION}

A bridge is a construction structure that functions to connect two parts of a road that are cut off by obstacles such as deep valleys, river courses, irrigation channels and dumps. This road that crosses the same plot and not others. Bridges are often a critical component of a road section, because as a determinant of the maximum load of vehicles passing through these roads [2]. This paper will show the calculation of the loading on the bridge structure according to [3] and [4]. Loads calculated are those that are used in accordance with the function of the bridge as a highway bridge. Charges are grouped into 3 load groups namely girder's own weight, additional dead load, and live load. For additional dead load analyzed, the load plate, asphalt, and diaphragm. As for the live load, that is D load which consists of distributed load (uniformly distributed load) and Knife Edge Load (KEL) load.

Calculation analysis of the two types of loading on the bridge structure using the same bridge modeling. The length of the girder is $40 \mathrm{~m}$, the distance between girders is $1.75 \mathrm{~m}$, and plate thickness is $0.2 \mathrm{~m}$. The compressive strength of concrete for girder is $40 \mathrm{MPa}$, while for pier and foundation it uses $35 \mathrm{MPa}$. The pre-stressed cable used is uncoated stress relieve 7 wires strand with an ultimate tensile strength of $18700 \mathrm{~kg} / \mathrm{cm}^{2}$, diameter of $12.7 \mathrm{~mm}$, effective area of $0.987 \mathrm{~cm}^{2}$, and modulus of elasticity $1.960 .000 \mathrm{~kg} / \mathrm{cm}^{2}$.

As for the purpose of this paper is as follows: (1) find out the results of a comparative analysis of the effect on the bridge girder; (2) find out the results of the analysis of influence on the bridge pillars; (3) find out the results of comparative analyses of effects on bridge abutments; (4) find out the results of analysis of influence on the bridge foundation.

\section{RESEARCH METHODOLOGY}

The burden on building structures is one of the most important things in building planning. Errors in load planning or application of loads in calculations will result in fatal errors in the results of the building design [5]. Based on this, it is important to update the loading regulations on this bridge. So the government issued a loading regulation on the latest bridge, SNI 1725-2016, to replace the RSNI T 022005. As for some differences between SNI 1725-2016 regulations with RSNI T 02-2005 in Table 1 and the research flow diagram in Figure 1 are as follows.

Table 1: Difference RSNI T 02-2005 and SNI 1725-2016

\begin{tabular}{|c|c|c|c|}
\hline No & Description & RSNI T 02-2005 & SNI $1725-2016$ \\
\hline 1 & $\begin{array}{l}\text { Lane load } \\
\text { "D" }\end{array}$ & & \\
\hline & $\begin{array}{l}\text { Load evenly } \\
\text { distributed }\end{array}$ & $8 \mathrm{kPa}$ & $9 \mathrm{kPa}$ \\
\hline & $\begin{array}{l}\text { Centered } \\
\text { line load }\end{array}$ & 44 kN/m & $49 \mathrm{kN} / \mathrm{m}$ \\
\hline & $\begin{array}{l}\text { Load } \\
\text { intensity }\end{array}$ & $\begin{array}{l}100 \% \text { load } \\
\text { intensity for line } \\
\text { width } \leq 5.5 \mathrm{~m} \\
\text { and additional } \\
\text { "D" loads must } \\
\text { be placed on the } \\
\text { remaining width } \\
\text { of tracks with } \\
50 \% \text { intensity. }\end{array}$ & $\begin{array}{l}\text { Distribution of } \\
\text { loads spread } \\
\text { across the entire } \\
\text { width of the } \\
\text { beam (not } \\
\text { including } \\
\text { parapets, curbs, } \\
\text { and sidewalks) } \\
\text { with 100\% } \\
\text { intensity. }\end{array}$ \\
\hline 2 & Truck load & & \\
\hline & $\begin{array}{l}\text { Truck load } \\
\text { plan }\end{array}$ & $450 \mathrm{kN}$ & $500 \mathrm{kN}$ \\
\hline
\end{tabular}


Fendy Rachmat et al., International Journal of Emerging Trends in Engineering Research, 8(9), September 2020, $5811-5818$

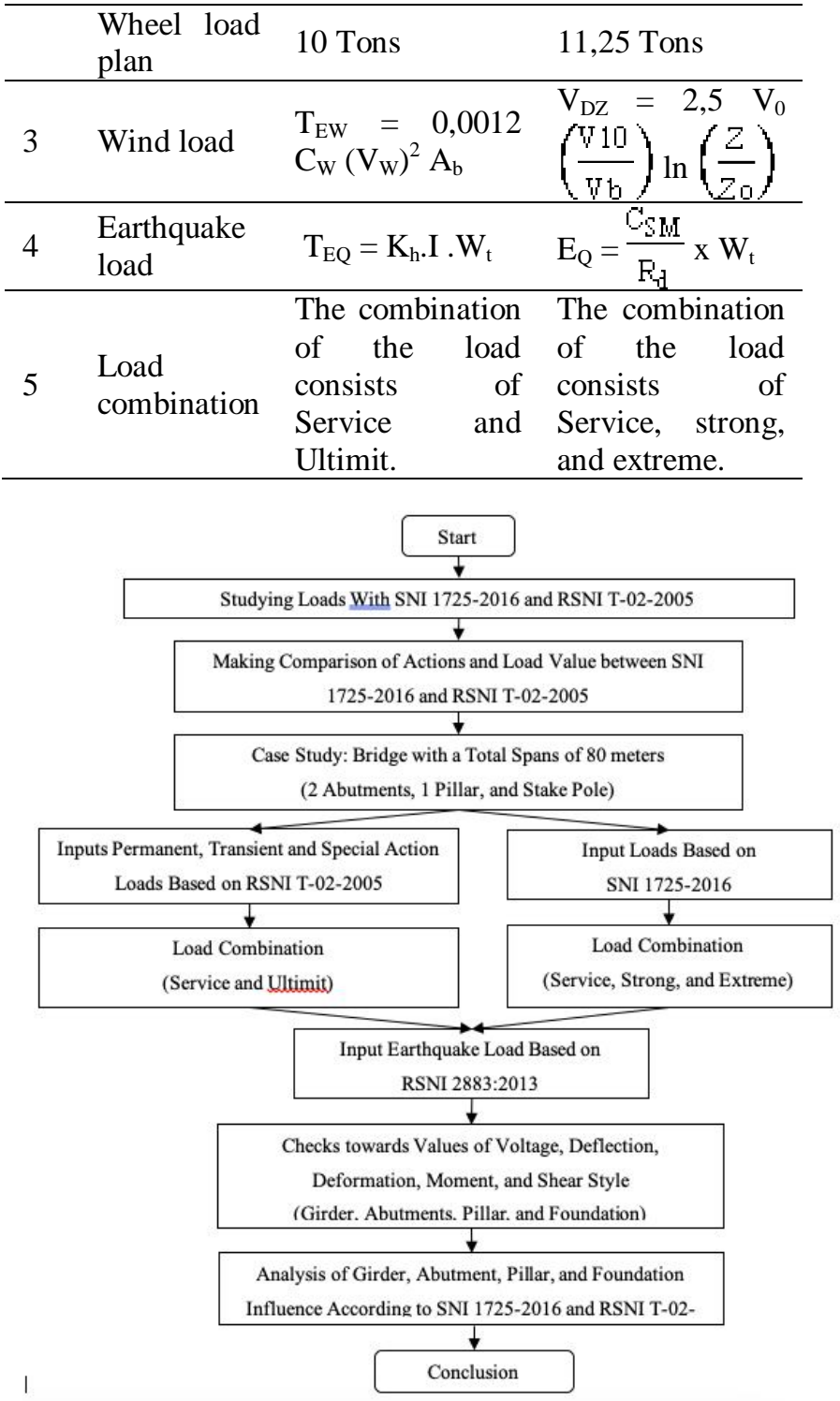

Figure 1: Research Flow Diagram

The factor of loading combination must be chosen in such a way as to produce extreme conditions due to the workload. For each combination of loading, the maximum and minimum drinking conditions must be investigated. The following is the combination of loading according to [3] regulations in Table 2 and the combination of loading according to SNI 1725-2016 in Table 3.

Table 2: Load Factor According to [3]

\begin{tabular}{|c|c|c|c|c|c|c|}
\hline $\begin{array}{l}\text { Combinat } \\
\text { ion }\end{array}$ & $\begin{array}{l}\text { Alon } \\
\mathrm{e} \\
\text { Weig } \\
\text { ht }\end{array}$ & $\begin{array}{l}\text { Additio } \\
\text { nal } \\
\text { Dead } \\
\text { Load }\end{array}$ & $\begin{array}{l}\text { Lan } \\
\text { e } \\
\text { Loa } \\
\text { d } \\
\text { "D" }\end{array}$ & $\begin{array}{l}\text { Bra } \\
\text { ke } \\
\text { Styl } \\
\text { e }\end{array}$ & $\begin{array}{l}\text { Wi } \\
\text { nd } \\
\text { Loa } \\
\text { d }\end{array}$ & $\begin{array}{l}\text { Earthqu } \\
\text { ake } \\
\text { Load }\end{array}$ \\
\hline Service 1 & 1 & 1 & 1 & 1 & - & - \\
\hline Service 2 & 1 & 1 & 0,5 & 0,5 & - & - \\
\hline Service 3 & 1 & 1 & 0,5 & 0,5 & 0,5 & - \\
\hline Ultimit 1 & 2 & 2 & 2 & 1 & 0,6 & - \\
\hline Ultimit 2 & 2 & 2 & 1 & 1 & - & - \\
\hline Ultimit 3 & 2 & 2 & 1 & 1 & 0,6 & - \\
\hline
\end{tabular}

\begin{tabular}{lllllll} 
Ultimit 4 & 2 & 2 & 1 & 1 & 1,2 & - \\
\hline $\begin{array}{l}\text { Ultimit } 5 \\
\text { Pontianak }\end{array}$ & 2 & 2 & - & - & - & 1 \\
\hline $\begin{array}{l}\text { Ultimit } 6 \\
\text { Jayapura }\end{array}$ & 2 & 2 & - & - & - & 1 \\
\hline
\end{tabular}

Table 3: Load Factor According to SNI 1725-2016

\begin{tabular}{|c|c|c|c|c|c|c|}
\hline $\begin{array}{l}\text { Combin } \\
\text { ation }\end{array}$ & $\begin{array}{l}\text { Alo } \\
\text { ne } \\
\text { Wei } \\
\text { ght }\end{array}$ & $\begin{array}{l}\text { Additio } \\
\text { nal } \\
\text { Dead } \\
\text { Load }\end{array}$ & $\begin{array}{l}\text { Lane } \\
\text { Load } \\
\text { "D" }\end{array}$ & $\begin{array}{l}\text { Bra } \\
\text { ke } \\
\text { Styl } \\
\text { e }\end{array}$ & $\begin{array}{l}\text { Win } \\
\text { d } \\
\text { Loa } \\
\text { d }\end{array}$ & $\begin{array}{l}\text { Earthqu } \\
\text { ake } \\
\text { Load }\end{array}$ \\
\hline $\begin{array}{l}\text { Service } \\
1\end{array}$ & 1 & 1 & 1 & 1 & 0,3 & - \\
\hline $\begin{array}{l}\text { Service } \\
2\end{array}$ & 1 & 1 & 1,3 & 0,3 & - & - \\
\hline $\begin{array}{l}\text { Service } \\
3\end{array}$ & 1 & 1 & 0,8 & 0,8 & - & - \\
\hline $\begin{array}{l}\text { Service } \\
4\end{array}$ & 1 & 1 & - & - & 0,7 & - \\
\hline Strong 1 & 1,2 & 2 & 1,8 & 1,8 & - & - \\
\hline Strong 2 & 1,2 & 2 & 1,4 & 1,4 & - & - \\
\hline Strong 3 & 1,2 & 2 & - & - & 0,4 & - \\
\hline $\begin{array}{l}\text { Extreme } \\
1 \\
\text { Pontiana } \\
\mathrm{k}\end{array}$ & 1,2 & 1,2 & 0,5 & 0,5 & - & 1 \\
\hline $\begin{array}{l}\text { Extreme } \\
1 \\
\text { Jayapura }\end{array}$ & 1,2 & 1,2 & 0,5 & 0,5 & - & 1 \\
\hline
\end{tabular}

\section{RESULT AND DISCUSSION}

The geometry of the bridge structure for calculation analysis is the same. With geometrical data the bridge structure is longitudinal is $40 \mathrm{~m}$ with transverse direction width of $9 \mathrm{~m}$, bridge height is $7 \mathrm{~m}$ and foundation depth is $10 \mathrm{~m}$. the number of girder I in the transversal direction is 5 units with a distance of girder $1.75 \mathrm{~m}$ and additional length at the left and right girder is $1 \mathrm{~m}$. Here is a picture of the geometry of the bridge structure (figure 2).

\subsection{Bridge Material Technical Specification}

Technical specifications of girders, pillars, foundations, and pre-stressed cables along with the arrangement of prestressed cable configurations as described in Tables 4-7. For the geometry of bridge structures found in Figure 2 below.

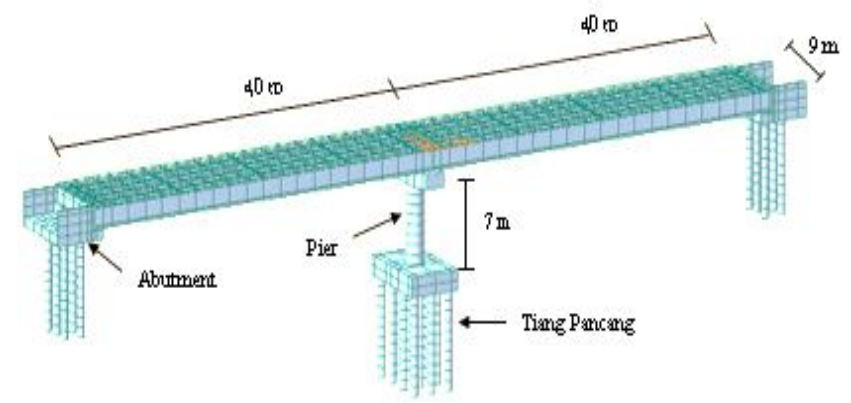

Figure 2: Geometry of Bridge Structures 
Fendy Rachmat et al., International Journal of Emerging Trends in Engineering Research, 8(9), September 2020,5811 - 5818

Table 4: Technical Specifications of Girder I and Pre-stressed Cables

\begin{tabular}{llll}
\hline Girder I & \multicolumn{3}{c}{ Pre-stressed Cable } \\
\hline Girder Height & $\begin{array}{l}2,3 \\
\mathrm{~m}\end{array}$ & $\begin{array}{l}\text { Nominal } \\
\text { Diameter }\end{array}$ & $12,7 \mathrm{~mm}$ \\
\hline $\begin{array}{l}\text { Distance between } \\
\text { Girders }\end{array}$ & $\begin{array}{l}1,75 \\
\mathrm{~m}\end{array}$ & Nominal Area & $\begin{array}{l}0,987 \\
\mathrm{~cm}^{2}\end{array}$ \\
\hline Plate Thickness & 20 & $\begin{array}{l}\text { Ultimate } \\
\text { Tensile }\end{array}$ & 18700 \\
& $\mathrm{~cm}$ & Strength & $\mathrm{kg} / \mathrm{cm}^{2}$ \\
\hline $\begin{array}{l}\text { Girder } \\
\text { Compressive }\end{array}$ & 40 & Low Relaxation & \\
Strength & $\mathrm{MPa}$ & & \\
\hline $\begin{array}{l}\text { Plate Compressive } \\
\text { Strength }\end{array}$ & $\begin{array}{l}20 \\
\mathrm{MPa}\end{array}$ & $\begin{array}{l}\text { Number of Pre- } \\
\text { Stressed Cables }\end{array}$ & 58 strand \\
\hline
\end{tabular}

Table 5: Bridge Pillar Technical Specifications

\begin{tabular}{|c|c|c|}
\hline Description & Value & Unit \\
\hline $\begin{array}{ll}\text { Concrete } & \text { Compressive } \\
\text { Strength }\left(f_{c}^{\prime}\right) & \\
\end{array}$ & 30 & $\mathrm{MPa}$ \\
\hline Concrete Elastic Modulus $\left(\mathrm{E}_{\mathrm{c}}\right)$ & 25742,96 & $\mathrm{MPa}$ \\
\hline Pier Height & 7 & $\mathrm{~m}$ \\
\hline Pier Diameter & 2 & $\mathrm{~m}$ \\
\hline Pier Head Width & 9 & $\mathrm{~m}$ \\
\hline
\end{tabular}

Table 6: Bridge Foundation Technical Specification

\begin{tabular}{|c|c|c|}
\hline Description & Value & Unit \\
\hline $\begin{array}{ll}\text { Concrete } & \text { Compressive } \\
\text { Strength }\left(f_{c}^{\prime}\right) & \\
\end{array}$ & 30 & Mpa \\
\hline Concrete Elastic Modulus $\left(\mathrm{E}_{\mathrm{c}}\right)$ & 25742,96 & $\mathrm{MPa}$ \\
\hline Foundation Length & 10 & $\mathrm{~m}$ \\
\hline Foundation Diameter & 0,8 & $\mathrm{~m}$ \\
\hline
\end{tabular}

Table 7:Pre-stressed Cable Configuration Arrangement

\begin{tabular}{llll}
\hline \multirow{2}{*}{$\begin{array}{l}\text { Tendon } \\
\text { Number }\end{array}$} & \multirow{2}{*}{$\begin{array}{l}\text { Number of } \\
\text { Strand }\end{array}$} & \multicolumn{2}{l}{ Strand Height From Below $(\mathrm{cm})$} \\
\cline { 3 - 4 } 1 & 15 & 1650 & Mdge \\
\hline 2 & 19 & 1350 & 200 \\
\hline 3 & 12 & 550 & 100 \\
\hline 4 & 12 & 300 & 100 \\
\hline
\end{tabular}

\subsection{Loads that Works}

Loads that work on the bridge according to [3] and [4]regulations as in Table 8 below.

Table 8: Loads that Works

\begin{tabular}{|c|c|c|c|}
\hline Load & $\begin{array}{l}\text { RSNI T } \\
(\mathrm{kN} / \mathrm{m})\end{array}$ & $\begin{array}{l}\text { SNI 1 } \\
(\mathrm{kN} / \mathrm{m})\end{array}$ & \\
\hline Alone Weight & 33,835 & 33,835 & 0 \\
\hline Additional Dead & 11,8 & 11,8 & 0 \\
\hline Brake Load & 50 & 27,06 & 45 \\
\hline Wind Load & 1,2 & 4,4 & 73 \\
\hline
\end{tabular}

\section{Lane Load}

\begin{tabular}{llll}
\hline Line Load & 48,4 & 68,6 & 30 \\
\hline $\begin{array}{l}\text { Loads Evenly } \\
\text { Divided }\end{array}$ & 7,7 & 11,03 & 30 \\
\hline $\begin{array}{l}\text { Earthquake } \\
\text { Loads }\end{array}$ & & \\
\hline $\begin{array}{l}\text { Pontianak } \\
\text { Jayapura }\end{array}$ & 2,35 & 2,35 & 0 \\
\hline
\end{tabular}

\subsection{Voltage Value Results}

In the discussion of the comparison of the value of the voltage on the girder will be obtained the results of the comparison of the maximum voltage value in the middle of the girder span based on calculations according to [3] and [4]. The following results are the comparison of the value of the voltage on the girder.

Table 9: Voltage Comparison in Girder

\begin{tabular}{|c|c|c|c|c|c|}
\hline \multirow[b]{2}{*}{ Situation } & \multirow{2}{*}{$\begin{array}{l}\text { Voltage } \\
\text { Limit } \\
\text { (Mpa) }\end{array}$} & \multicolumn{2}{|c|}{ RSNI T 02-2005 } & \multicolumn{2}{|c|}{ SNI 1725-2016 } \\
\hline & & $\begin{array}{l}\text { Voltage } \\
\text { (Mpa) }\end{array}$ & Check & $\begin{array}{l}\text { Voltage } \\
\text { (Mpa) }\end{array}$ & Check \\
\hline \multicolumn{6}{|c|}{$\begin{array}{l}\text { Initial } \\
\text { Condition } \\
\end{array}$} \\
\hline $\begin{array}{l}\text { Upper } \\
\text { Fiber }\end{array}$ & 2,82 & 2,56 & OK & 2,56 & $\mathrm{OK}$ \\
\hline $\begin{array}{l}\text { Fiber } \\
\text { Bottom }\end{array}$ & $-19,2$ & $-11,28$ & OK & $-11,28$ & OK \\
\hline \multicolumn{6}{|c|}{$\begin{array}{l}\text { Service } \\
\text { Conditions } \\
\end{array}$} \\
\hline $\begin{array}{l}\text { Upper } \\
\text { Fiber }\end{array}$ & -18 & $-6,5$ & OK & $-7,6$ & OK \\
\hline $\begin{array}{l}\text { Fiber } \\
\text { Bottom }\end{array}$ & 3,16 & 1,6 & $\mathrm{OK}$ & 2,5 & $\mathrm{OK}$ \\
\hline
\end{tabular}

\subsection{Deflection Analysis Results in Girder}

In this sub chapter will explain the stress value that occurs in the middle span due to axial force, latitude, bending moment and torque which have been analyzed with the help of the Midas civil program. The following results of deflection values on the girder reviewed in the middle of the span according to [3] and [4].

Table 10: Deflection Value of RSNI T 02-2005

\begin{tabular}{|c|c|c|c|c|c|}
\hline $\mathrm{x}$ & $\begin{array}{l}\text { Lane } \\
\text { "D" }\end{array}$ & Load & Service 1 & Service 2 & Service 3 \\
\hline 0 & 0 & & 0 & 0 & 0 \\
\hline 2 & $-1,1$ & & $-6,1$ & $-5,4$ & $-4,9$ \\
\hline 4 & $-2,1$ & & $-9,2$ & $-7,8$ & $-7,1$ \\
\hline 6 & $-2,9$ & & $-12,1$ & $-10,2$ & $-9,5$ \\
\hline 8 & $-3,7$ & & $-14,7$ & $-12,2$ & $-11,6$ \\
\hline 10 & $-4,3$ & & $-16,1$ & $-14,1$ & $-13,3$ \\
\hline 12 & $-4,9$ & & $-17,2$ & $-15,3$ & $-14,9$ \\
\hline 14 & $-5,4$ & & $-18,4$ & $-16,5$ & $-16,1$ \\
\hline 16 & $-5,7$ & & $-19,3$ & $-17,1$ & $-16,7$ \\
\hline
\end{tabular}


Fendy Rachmat et al., International Journal of Emerging Trends in Engineering Research, 8(9), September 2020, $5811-5818$

\begin{tabular}{lllll}
18 & $-5,9$ & $-20,2$ & $-17,6$ & $-17,1$ \\
\hline 20 & $-6,1$ & $-20,7$ & $-17,8$ & $-17,4$ \\
\hline
\end{tabular}

Table 11: Deflection Value of SNI 1725-2016

\begin{tabular}{|c|c|c|c|c|c|}
\hline $\mathrm{x}$ & $\begin{array}{l}\text { Lane Load } \\
\text { "D" }\end{array}$ & $\begin{array}{l}\text { Service } \\
1\end{array}$ & $\begin{array}{l}\text { Service } \\
2\end{array}$ & $\begin{array}{l}\text { Service } \\
3\end{array}$ & $\begin{array}{l}\text { Service } \\
4\end{array}$ \\
\hline 0 & 0 & 0 & 0 & 0 & 0 \\
\hline 2 & $-3,1$ & $-7,6$ & $-8,5$ & $-7,1$ & $-4,5$ \\
\hline 4 & $-5,3$ & $-11,9$ & $-13,5$ & $-10,8$ & $-6,5$ \\
\hline 6 & $-7,5$ & $-15,9$ & $-18,2$ & $-14,2$ & $-8,9$ \\
\hline 8 & $-9,5$ & $-19,6$ & $-22,4$ & $-17,7$ & $-10,1$ \\
\hline 10 & $-11,3$ & $-22,8$ & $-26,7$ & $-20,4$ & $-11,1$ \\
\hline 12 & $-12,8$ & $-25,3$ & $-29,1$ & $-22,7$ & $-12,4$ \\
\hline 14 & $-14,3$ & $-27,2$ & $-31,4$ & $-24,4$ & $-13,1$ \\
\hline 16 & $-15,2$ & $-28,5$ & $-33,2$ & $-25,5$ & $-13,5$ \\
\hline 18 & $-15,7$ & $-29,2$ & $-33,8$ & $-26,1$ & $-13,8$ \\
\hline 20 & $-16,1$ & $-29,8$ & $-34,2$ & $-26,8$ & $-14,2$ \\
\hline
\end{tabular}

3.5 Result of Moment Value and Shear Style

In the sub chapter comparison of the moment value and shear style in the girder will be obtained the results of the comparison of the maximum moment value in the middle of the girder span based on calculations according to [3] and [4]. The results of the comparison of moment and shear style values as shown in Table 12 to Table 15 below. 
Table 12: Moment Value of RSNI T 02-2005

\begin{tabular}{|c|c|c|c|c|c|c|c|c|c|c|c|}
\hline \multirow{2}{*}{ Load } & \multicolumn{11}{|c|}{ Span (m) } \\
\hline & 0 & 2 & 4 & 6 & 8 & 10 & 12 & 14 & 16 & 18 & 20 \\
\hline Alone Weight & 0 & 1609,3 & 3088,2 & 4397,5 & 5537,2 & 6507,4 & 7284,3 & 7891,6 & 8328,8 & 8506,1 & 8694,2 \\
\hline Additional Dead & 0 & 275,2 & 713,1 & 874,9 & 998,6 & 1138,3 & 1240,3 & 1304,2 & 1315,6 & 1320,4 & 1330,9 \\
\hline Lane Load "D" & 0 & 370,2 & 556,1 & 737,2 & 914,5 & 1002,6 & 1085,4 & 1163,2 & 1237,2 & 1306,5 & 1306,7 \\
\hline Brake Load & 0 & 4,6 & 9,2 & 13,7 & 18,3 & 22,9 & 27,5 & 32,1 & 36,6 & 41,2 & 45,8 \\
\hline Wind Load & 0 & 36,6 & 54,9 & 73,2 & 91,5 & 109,7 & 128,0 & 146,3 & 164,6 & 182,9 & 201,2 \\
\hline $\begin{array}{l}\text { Pontianak } \\
\text { Earthquake }\end{array}$ & 0 & 432,0 & 647,9 & 863,9 & 1079,9 & 1295,9 & 1511,9 & 1727,9 & 1943,8 & 2159,8 & 2375,8 \\
\hline $\begin{array}{l}\text { Jayapura } \\
\text { Earthquake }\end{array}$ & 0 & 1591,6 & 2387,4 & 3183,2 & 3979,0 & 4774,9 & 5570,7 & 6366,5 & 7162,3 & 7958,1 & 8753,9 \\
\hline Ultimit 1 & 0 & 2904,7 & 5635,8 & 7635,4 & 9381,7 & 10812,1 & 11963,2 & 12858,5 & 13471,0 & 13801,1 & 14015,2 \\
\hline Ultimit 2 & 0 & 2534,5 & 5079,7 & 6898,2 & 8467,2 & 9809,5 & 10877,8 & 11695,3 & 12233,8 & 12494,6 & 12708,5 \\
\hline Ultimit 3 & 0 & 2556,4 & 5112,6 & 6942,1 & 8522,1 & 9875,3 & 10954,6 & 11783,1 & 12332,6 & 12604,4 & 12829,2 \\
\hline Ultimit 4 & 0 & 2578,4 & 5145,5 & 6986,0 & 8577,0 & 9941,2 & 11031,4 & 11870,9 & 12431,4 & 12714,1 & 12949,9 \\
\hline Ultimit 4 Pontianak & 0 & 2591,7 & 5162,3 & 7011,2 & 8614,3 & 10079,9 & 11276,8 & 12227,9 & 12903,8 & 13306,7 & 13731,8 \\
\hline Ultimit 4 Jayapura & 0 & 3751,3 & 6901,8 & 9330,5 & 11513,4 & 13558,9 & 15335,6 & 16866,5 & 18122,3 & 19105,0 & 20109,9 \\
\hline
\end{tabular}

Table 13: Shear Style Value of RSNI T 02-2005

\begin{tabular}{|c|c|c|c|c|c|c|c|c|c|c|c|}
\hline \multirow{2}{*}{ Load } & \multicolumn{11}{|c|}{ Span (m) } \\
\hline & 0 & 2 & 4 & 6 & 8 & 10 & 12 & 14 & 16 & 18 & 20 \\
\hline Alone Weight & 835,1 & 824,2 & 781,9 & 697,1 & 612,3 & 527,5 & 430,7 & 345,9 & 261,1 & 174,5 & 0,0 \\
\hline Additional Dead & 149,8 & 128,5 & 109,5 & 90,4 & 81,4 & 79,6 & 60,3 & 41,5 & 22,4 & 15,7 & 0,0 \\
\hline Lane Load "D" & 143,7 & 115,5 & 100,9 & 95,8 & 87,3 & 81,4 & 66,2 & 50,6 & 35,2 & 19,8 & 22,0 \\
\hline Brake Load & 0,9 & 0,9 & 0,9 & 0,9 & 0,9 & 0,9 & 0,9 & 0,9 & 0,9 & 0,9 & 0,9 \\
\hline Wind Load & 20,2 & 18,4 & 16,5 & 14,7 & 12,9 & 11,0 & 9,2 & 7,3 & 5,5 & 3,7 & 1,8 \\
\hline Pontianak Earthquake & 23,6 & 21,4 & 19,3 & 17,1 & 15,0 & 12,9 & 10,7 & 8,6 & 6,4 & 4,3 & 2,1 \\
\hline Jayapura Earthquake & 107,2 & 97,2 & 87,2 & 77,2 & 67,2 & 57,2 & 45,0 & 38,3 & 21,8 & 10,3 & 0,3 \\
\hline Ultimit 1 & 2270,2 & 2148,3 & 1995,4 & 1776,3 & 1570,6 & 1384,5 & 1120,8 & 881,3 & 641,6 & 423,1 & 46,0 \\
\hline Ultimit 2 & 2114,4 & 2021,8 & 1884,6 & 1671,7 & 1475,6 & 1296,5 & 1049,1 & 826,3 & 603,1 & 401,1 & 22,9 \\
\hline Ultimit 3 & 2126,5 & 2032,8 & 1894,5 & 1680,5 & 1483,3 & 1303,1 & 1054,6 & 830,7 & 606,4 & 403,3 & 24,0 \\
\hline Ultimit 4 & 2138,6 & 2043,8 & 1904,4 & 1689,3 & 1491,0 & 1309,7 & 1060,1 & 835,1 & 609,7 & 405,5 & 25,1 \\
\hline Ultimit 4 Pontianak & 1993,4 & 1926,8 & 1802,1 & 1592,1 & 1402,4 & 1227,1 & 992,7 & 783,4 & 573,4 & 384,7 & 2,1 \\
\hline Ultimit 4 Jayapura & 2077,0 & 2002,6 & 1870,0 & 1652,2 & 1454,6 & 1271,4 & 1027,0 & 813,1 & 588,8 & 390,7 & 0,3 \\
\hline
\end{tabular}


Table 14: Moment Value of RSNI T 02-2005

\begin{tabular}{|c|c|c|c|c|c|c|c|c|c|c|c|}
\hline \multirow{2}{*}{ Load } & \multicolumn{11}{|c|}{ Span $(\mathrm{m})$} \\
\hline & 0 & 2 & 4 & 6 & 8 & 10 & 12 & 14 & 16 & 18 & 20 \\
\hline Alone Weight & 0 & 1609,3 & 3088,2 & 4397,5 & 5537,2 & 6507,4 & 7284,3 & 7891,6 & 8328,8 & 8506,1 & 8694,2 \\
\hline Additional Dead & 0 & 275,2 & 713,1 & 874,9 & 998,6 & 1138,3 & 1240,3 & 1304,2 & 1315,6 & 1320,4 & 1330,9 \\
\hline Lane Load "D" & 0 & 488,1 & 931,5 & 1331,0 & 1686,4 & 1997,8 & 2264,1 & 2486,3 & 2664,7 & 2799,2 & 2889,3 \\
\hline Brake Load & 0 & 5,1 & 7,6 & 10,1 & 12,7 & 15,2 & 17,7 & 20,3 & 22,8 & 25,3 & 27,9 \\
\hline Wind Load & 0 & 91,4 & 137,1 & 182,9 & 228,6 & 274,3 & 320,0 & 365,7 & 411,4 & 457,1 & 502,9 \\
\hline Pontianak Earthquake & 0 & 432,0 & 647,9 & 863,9 & 1079,9 & 1295,9 & 1511,9 & 1727,9 & 1943,8 & 2159,8 & 2375,8 \\
\hline Jayapura Earthquake & 0 & 1591,6 & 2387,4 & 3183,2 & 3979,0 & 4774,9 & 5570,7 & 6366,5 & 7162,3 & 7958,1 & 8753,9 \\
\hline Strong I & 0 & 2403,7 & 4969,5 & 6802,3 & 8377,8 & 9804,4 & 10958,5 & 11855,2 & 12466,0 & 12828,6 & 13129,2 \\
\hline Strong II & 0 & 2206,4 & 4593,9 & 6265,9 & 7698,2 & 8999,2 & 10045,7 & 10852,6 & 11391,0 & 11698,8 & 11962,4 \\
\hline Strong III & 0 & 1552,6 & 3334,0 & 4461,4 & 5410,9 & 6290,8 & 6979,2 & 7489,6 & 7793,0 & 7927,3 & 8079,5 \\
\hline Extreme 1 Pontianak & 0 & 1976,9 & 3829,9 & 5227,9 & 6456,4 & 7580,4 & 8520,6 & 9291,3 & 9875,0 & 10272,9 & 10661,9 \\
\hline Extreme 1 Jayapura & 0 & 3131,5 & 5561,8 & 7537,1 & 9342,9 & 11044,2 & 12561,7 & 13909,6 & 15070,6 & 16045,8 & 17012,2 \\
\hline
\end{tabular}

Table 15: Shear Style Value of SNI 1725-201

\begin{tabular}{|c|c|c|c|c|c|c|c|c|c|c|c|}
\hline \multirow{2}{*}{ Load } & \multicolumn{11}{|c|}{ Span $(\mathrm{m})$} \\
\hline & 0 & 2 & 4 & 6 & 8 & 10 & 12 & 14 & 16 & 18 & 20 \\
\hline Alone Weight & 835,1 & 824,2 & 781,9 & 697,1 & 612,3 & 527,5 & 430,7 & 345,9 & 261,1 & 174,5 & 0,0 \\
\hline Additional Dead & 149,8 & 128,5 & 109,5 & 90,4 & 81,4 & 79,6 & 60,3 & 41,5 & 22,4 & 15,7 & 0,0 \\
\hline Lane Load "D" & 258,5 & 232,7 & 210,7 & 188,7 & 166,5 & 144,2 & 122,2 & 100,1 & 78,2 & 56,2 & 34,2 \\
\hline Brake Load & 1,5 & 1,5 & 1,5 & 1,5 & 1,5 & 1,5 & 1,5 & 1,5 & 1,5 & 1,5 & 1,5 \\
\hline Wind Load & 50,3 & 45,7 & 41,2 & 36,6 & 32,0 & 27,4 & 22,9 & 18,3 & 13,7 & 9,1 & 4,6 \\
\hline Pontianak Earthquake & 23,6 & 21,4 & 19,3 & 17,1 & 15,0 & 12,9 & 10,7 & 8,6 & 6,4 & 4,3 & 2,1 \\
\hline Jayapura Earthquake & 107,2 & 97,2 & 87,2 & 77,2 & 67,2 & 57,2 & 45,0 & 38,3 & 21,8 & 10,3 & 0,3 \\
\hline Strong I & 1769,7 & 1667,6 & 1539,2 & 1359,7 & 1200,0 & 1054,5 & 860,1 & 681,0 & 501,6 & 344,7 & 64,3 \\
\hline Strong II & 1665,7 & 1573,9 & 1454,4 & 1283,6 & 1132,8 & 996,2 & 810,6 & 640,3 & 469,7 & 321,6 & 50,0 \\
\hline Strong III & 1321,8 & 1264,3 & 1173,7 & 1032,0 & 910,4 & 803,2 & 646,6 & 505,4 & 363,6 & 244,5 & 1,8 \\
\hline Extreme 1 Pontianak & 1335,4 & 1281,8 & 1195,1 & 1057,2 & 931,4 & 814,2 & 661,8 & 524,2 & 386,5 & 261,4 & 20,0 \\
\hline Extreme 1 Jayapura & 1419,1 & 1357,6 & 1263,0 & 1117,3 & 983,7 & 858,6 & 696,1 & 553,9 & 401,8 & 267,3 & 18,1 \\
\hline
\end{tabular}


Fendy Rachmat et al., International Journal of Emerging Trends in Engineering Research, 8(9), September 2020, $5811-5818$

\subsection{The Value of Inner Style on the Pillar}

In this sub chapter will explain the results of the ultimate load on the pillars and bridge abutments resulting from a combination of loading according to [3] and [4]regulations. Following are the results of the calculation of the value of internal forces on the pillars and bridge abutments which can be shown in Table 12 to Table 15 .

Table 16: Inner Styles on Pillars of RSNI T 02-2005

\begin{tabular}{|c|c|c|c|c|}
\hline No & Combination & $\begin{array}{l}\text { Axial } \\
\text { Load } \\
\mathrm{P}(\mathrm{kN})\end{array}$ & $\begin{array}{l}\text { M } \\
\text { Moment } \\
(\mathrm{kN})\end{array}$ & $\begin{array}{l}\text { Horizontal } \\
\text { Style } \\
\mathrm{T}(\mathrm{kN} . \mathrm{m})\end{array}$ \\
\hline 1 & Ultimit 1 & 16234,8 & 1367,6 & 433,45 \\
\hline 2 & Ultimit 2 & 15387,2 & 1035,8 & 80,2 \\
\hline 3 & Ultimit 3 & 15298,5 & 1367,5 & 333,4 \\
\hline 4 & Ultimit 4 & 15298,5 & 1835,1 & 566,9 \\
\hline 5 & $\begin{array}{ll}\text { Ultimit } & 5 \\
\text { Jayapura }\end{array}$ & 14128,1 & 8360,9 & 7028,7 \\
\hline 6 & $\begin{array}{ll}\text { Ultimit } & 5 \\
\text { Pontianak } & \end{array}$ & 14128,1 & 1337,4 & 1123,5 \\
\hline
\end{tabular}

Table 17: Inner Styles on Pillars of SNI 1725-2016

\begin{tabular}{lllll}
\hline No & Combination & $\begin{array}{l}\text { Axial } \\
\text { Load } \\
\mathrm{P}(\mathrm{kN})\end{array}$ & $\begin{array}{l}\text { M } \\
\text { Moment } \\
(\mathrm{kN})\end{array}$ & $\begin{array}{l}\text { Horizontal } \\
\text { Style } \\
\mathrm{T}(\mathrm{kN} . \mathrm{m})\end{array}$ \\
\hline 1 & Strong I & 18705,5 & 986,2 & 543,5 \\
\hline 2 & Strong II & 18705,5 & 635,7 & 389,4 \\
\hline 3 & Strong III & 14128,1 & 754,6 & 130,1 \\
\hline 4 & $\begin{array}{l}\text { Jayapura } \\
\text { Extreme }\end{array}$ & 14333,6 & 9234,5 & 8475,2 \\
\hline 5 & $\begin{array}{l}\text { Pontianak } \\
\text { Extreme }\end{array}$ & 14333,6 & 1458,4 & 2223,7 \\
\hline
\end{tabular}

Table 18: Inner Styles on Abutments of RSNI T 02-2005

\begin{tabular}{|c|c|c|c|c|}
\hline No & Combination & $\begin{array}{l}\text { Axial } \\
\text { Load } \\
\mathrm{P}(\mathrm{kN}) \\
\end{array}$ & $\begin{array}{l}\text { M } \\
\text { Moment } \\
(\mathrm{kN})\end{array}$ & $\begin{array}{l}\text { Horizontal } \\
\text { Style } \\
\mathrm{T}(\mathrm{kN} . \mathrm{m})\end{array}$ \\
\hline 1 & Ultimit 1 & 8117,4 & 633,78 & 266,72 \\
\hline 2 & Ultimit 2 & 7693,6 & 517,9 & 40,1 \\
\hline 3 & Ultimit 3 & 7649,25 & 633,7 & 166,7 \\
\hline 4 & Ultimit 4 & 7649,25 & 1067,5 & 273,45 \\
\hline 5 & $\begin{array}{ll}\text { Ultimit } & 5 \\
\text { Jayapura }\end{array}$ & 7064,05 & 4180,4 & 3014,35 \\
\hline 6 & $\begin{array}{ll}\text { Ultimit } & 5 \\
\text { Pontianak }\end{array}$ & 7064,05 & 668,7 & 561,75 \\
\hline
\end{tabular}

Table 19: Inner Styles on Abutments of SNI 1725-2016

\begin{tabular}{lllll}
\hline No & Combination & $\begin{array}{l}\text { Axial } \\
\text { Load } \\
\mathrm{P}(\mathrm{kN})\end{array}$ & $\begin{array}{l}\text { M } \\
\text { Mome } \\
\mathrm{nt}(\mathrm{kN})\end{array}$ & $\begin{array}{l}\text { Horizontal } \\
\text { Style } \\
\mathrm{T}(\mathrm{kN} . \mathrm{m})\end{array}$ \\
\hline 1 & Strong I & 9352,75 & 580,1 & 271,75 \\
\hline 2 & Strong II & 9352,75 & 317,85 & 194,7 \\
\hline 3 & Strong III & 7064,05 & 377,3 & 75,05 \\
\hline 4 & $\begin{array}{l}\text { Jayapura } \\
\text { Extreme }\end{array}$ & 7166,8 & $\begin{array}{l}4617,2 \\
5\end{array}$ & 4237,6 \\
\hline \multirow{2}{*}{5} & $\begin{array}{l}\text { Pontianak } \\
\text { Extreme }\end{array}$ & 7166,8 & 729,2 & 1121,85 \\
\hline
\end{tabular}

\subsection{The Value of Inner Styles on the Foundation}

In this sub chapter will explain the ultimate value of load that occurs on the foundation due to the axial force and moment style that have been analyzed with the help of the Midas civil program. The following results are the value of ultimate load on the foundation according to [3] and [4].

Table 20: Inner Styles on the Foundation of RSNI T 02-2005

\begin{tabular}{|c|c|c|c|c|}
\hline $\begin{array}{l}\mathrm{N} \\
\mathrm{o}\end{array}$ & Combination & $\begin{array}{l}\text { Axial } \\
\text { Load } \\
\text { V }(k N)\end{array}$ & $\begin{array}{l}\mathrm{M}_{\mathrm{x}} \\
\text { Moment } \\
\text { (kN.m) }\end{array}$ & $\begin{array}{l}\mathrm{M}_{\mathrm{y}} \\
\text { Moment }(\mathrm{kN} \text {. } \\
\mathrm{m})\end{array}$ \\
\hline 1 & Ultimit 1 & 16234,8 & 922,4 & 97,4 \\
\hline 2 & Ultimit 2 & 15387,2 & 922,4 & 97,4 \\
\hline 3 & Ultimit 3 & 15298,5 & 922,4 & 97,4 \\
\hline 4 & Ultimit 4 & 15298,5 & 922,4 & 196,6 \\
\hline 5 & $\begin{array}{ll}\text { Ultimit } & 5 \\
\text { Jayapura } & \end{array}$ & 14128,1 & 3795,6 & 4871,6 \\
\hline 6 & $\begin{array}{ll}\text { Ultimit } & 5 \\
\text { Pontianak } & \\
\end{array}$ & 14128,1 & 1892,7 & 2145,9 \\
\hline
\end{tabular}

Table 21: Inner Styles on the Foundation of SNI 1725-2016

\begin{tabular}{lllll}
\hline $\mathrm{N}$ & Combination & $\begin{array}{l}\text { Axial } \\
\text { Load } \\
\mathrm{V}(\mathrm{kN})\end{array}$ & $\begin{array}{l}\mathrm{M}_{\mathrm{x}} \\
\text { Moment } \\
(\mathrm{kN} . \mathrm{m})\end{array}$ & $\begin{array}{l}\mathrm{M}_{\mathrm{y}} \\
\text { Moment }(\mathrm{kN} .\end{array}$ \\
\hline 1 & Strong I & 18705,5 & 786,9 & 126,3 \\
\hline 2 & Strong II & 18705,5 & 573,2 & 126,3 \\
\hline 3 & Strong III & 14128,1 & 131,5 & 396,3 \\
\hline 4 & $\begin{array}{l}\text { Jayapura } \\
\text { Extreme }\end{array}$ & 14333,6 & 5693,4 & 7307,4 \\
\hline 5 & $\begin{array}{l}\text { Pontianak } \\
\text { Extreme }\end{array}$ & 14333,6 & 2271,24 & 2575,08 \\
\hline
\end{tabular}

\section{CONCLUSION AND SUGGESTION}

From the analysis conducted on the bridge structure, can be concluded several things as follows:

a. The value of the style in shear and moment due to the "D" lane load for SNI 1725-2016 is greater than RSNI $\mathrm{T}$ 02-2005. However, due to the brake load at SNI 1725-2016 is lower than RSNI T 02-2005;

b. Deflection value due to vehicle life load for SNI 17252016 is greater than RSNI T 02-2005. This is because the distribution factor of the "D" lane load in SNI 17252016 is greater;

c. The style on the bridge pillar in SNI 1725-2016 is greater than that of the 02N 2005 RSNI. An increase in axial style, moment style, and horizontal style;

d. The style on the bridge foundation on SNI 1725-2016 is greater than that of RSNI T 02-2005. An increase in axial style, moment style, and horizontal style;

e. The value of voltage and deflections in SNI 1725-2016 is an increase compared to RSNI regulation T 02-2005, but the voltage value is still below its permit limit value.

Based on the results of the analysis of this study, then to get better results, the authors provide the following advice: 
Fendy Rachmat et al., International Journal of Emerging Trends in Engineering Research, 8(9), September 2020, $5811-5818$

a. For further analysis it is necessary to review for bridges that have a span of more than $40 \mathrm{~m}$, looking at the results of the voltage value on the bridge which is approaching the value of the voltage permit limit;

b. Henceforth it needs to be reviewed for bridges in addition to using girder I.

\section{REFERENCES}

[1] B. S. Nasional, RSNI 2833:2013 Perancangan Jembatan Terhadap Beban Gempa. Jakarta: Badan Standarisasi Nasional, 2013.

[2] H.J.Sturyk, K. H. J. W. Van Der Ven,\& SoemargonoJembatan Versi Bahasa Indonesia. Jakarta: PT. Pradnya Paramita, 1984.

[3] B. S. Nasional, RSNI T-02-2005 Pembebanan Untuk Jembatan. Jakarta: Badan Standarisasi Nasional, 2005.

[4] B. S. Nasional, SNI 1725-2016 Pembebanan Untuk Jembatan. Jakarta: Badan Standarisasi Nasional, 2016.

[5] T. P. Winarti, "Beban Struktur Bangunan," 2016. [Online].

Available:

https://ceritatitinpw.wordpress.com/2016/03/27/bebanstruktur-bangunan/. [Accessed: 09-Jul-2018].

[6] O. O.Gorshkova, Construction of Long-Distance Oil Pipelines, International Journal of Emerging Trends in Engineering Research, Volume 8, No. 1, 2020 